\title{
Impact De La Volatilité De Change Sur La Performance Financière Des Entreprises Cotées
}

\author{
El Mehdi Mrhari, Doctorant \\ Institut supérieur de Commerce et d'Administration \\ des Entreprises (ISCAE), Maroc \\ Driss Daoui, Docteur \\ Université Ibn Tofail, Maroc
}

Doi:10.19044/esj.2018.v14n19p1 ～URL:http://dx.doi.org/10.19044/esj.2018.v14n19p1

\begin{abstract}
The main objective of this article is to measure and analyze the exchange rate volatility impact on the financial performance of Moroccan companies listed on Casablanca Stock Exchange during the last decade. We used linear models to study a sample of 56 companies.

This paper prove the link between Dirham volatility and the companies financial performance, however this relationship was weak. In addition, we discussed the causes of this phenomenon and its managerial implications.
\end{abstract}

Keywords : Exchange rate volatility, Financial performance, International trade, Currency risk.

\section{Résumé}

L'objectif principal de cet article est de mesurer et d'analyser l'effet de la volatilité de change sur la performance financière des entreprises marocaines cotées à la Bourse de Casablanca au cours de la dernière décennie. Nous avons utilisé des modèles linéaires pour étudier un échantillon de 56 entreprises.

Cet article montre le lien entre la volatilité du Dirham et la performance financière des entreprises cependant cette relation était faible. En outre, nous avons discuté des causes de ce phénomène et ses implications managériales.

Mots clés : Volatilité de change, Performance financière, Commerce internationale, Risque de change.

\section{Introduction}

A l'heure actuelle, la mondialisation vient de submerger les frontières monétaires entre nations ce qui a rendu l'agent économique plus vulnérable 
aux chocs externes. Par ailleurs, le bouleversement de l'ère de l'accord Bretton Woods en 1976 a mis fin à la fixité des taux de change. Ainsi la migration accrue des capitaux et l'internationalisation massive des entreprises ont multiplié les points de contact de l'investisseur avec d'autres monnaies.

Par conséquent, plusieurs problèmes d'ordre comptable ont vu le jour car malgré la pertinence des normes en la matière elles ne permettent pas l'enregistrement parfait des retombés de la volatilité de change surtout lorsque il s'agit de l'exposition économique Häberle (1999) ${ }^{1}$, Alaoui Ismailiet M'kaddem (2009) ${ }^{2}$. Et d'autre part d'ordre financier puisque la rentabilité de la société est susceptible d'être menacée d'où vient l'intérêt de cet article qui est le premier en son genre au Maroc à aborder l'effet des variations de change en se basant sur des données comptables d'entreprises.

A travers ce papier, nous aurons l'occasion de rependre à cette problématique «Quel est l'impact de la volatilité du Dirham sur la performance financière de l'entreprise ? ».Pour appréhender la performance financière nous avons fait appel à trois indicateurs : le résultat courant, le besoin en fonds de roulement et les charges de personnel. Quant à la période d'analyse s'étale principalement de 2006 à 2016 ou à nouveau ordre monétaires mondial s'est établi avec l'avènement de l'Euro et la montée des pays asiatiques.

En sus, ce travail donnera aux investisseurs une vision globale sur le mode de management du risque de change au Maroc ainsi il facilitera la compréhension des inducteurs de la performance des entreprises nationales qu'elles soient exportatrices, importatrices ou domestiques. En effet, l'élargissement du champ d'étude nous procure une particularité non négligeable.

Dans cet article, nous nous focaliserons sur le cas du Dirham «la Monnaie du Maroc » pour plusieurs raisons : Premièrement, le caractère de monnaie exotique que détient cette devise sachant que les études sur ce genre de monnaie sont rares. Deuxièmement, la volonté de la banque centrale de libéraliser le Dirham offre un contexte propice pour évaluer la pertinence d'un basculement vers un régime flottant. Troisièmement, l'état de l'économie marocaine qui se répertorie dans la catégorie des pays en voie de développement ou la compétitivité est basée essentiellement sur les prix.

\section{Revue de la littérature}

La volatilité est un concept polysémique qui change de connotation d'une discipline à une autre en effet nous avons adopté la définition

\footnotetext{
${ }^{1}$ Risque de change, entreprise et investisseur. Le cas du marché suisse des actions/ Rainer Häberle P 34.

${ }^{2}$ Variations des taux de change et facteurs de sensibilité des entreprises exportatrices marocaines / Alaoui Ismaili et M'kaddem P 39.
} 
de Marston (1988) «There are two types of exchange rate variability, volatility and misalignment. Volatility is the day-to-day, month-to-month variability of exchange rates. Misalignment, in contrast, is the persistent departure of an exchange rate fromits long run competitive level. ${ }^{3}$ »En d'autres termes, la volatilité de change mesure l'ampleur de la variation du taux de change au fil du temps.

Théoriquement, cette instabilité de change n'est pas sans incidences sur l'entreprise. D'ailleurs, plusieurs chercheurs se sont penchés sur cette question en utilisant divers variables proxies. En guise d'exemple, Martin et Mauer (2003) ont manié le résultat d'exploitation qui leurs a permis de relever 91\% d'exportateurs américains menacés par la volatilité de change. De l'autre côté, Bartram (2008) a régressé le bénéfice par action sur les variations de change ainsi il a remarqué un effet contemporain minime juste $8.4 \%$ des entreprises non financières en revanche à long terme la relation est plus importante $46.1 \%$.

Par ailleurs, Krapl et O'Brien (2015) ont étudié également le bénéfice par action de 3659 sociétés cependant les résultats n'étaient pas très encourageants puisque seulement $18 \%$ des entreprises sont touchées avec des coefficients de sensibilité relativement minces « entre -0.075 et 0.289 ».

Il faut rappeler que le signe de ces coefficients varie en fonction de la nature des activités de la firme. Etant donné que les entreprises exportatrices possèdent un paramètre positif contrairement aux sociétés importatrices qui ont des coefficients de sensibilité négatifs « au cotation certaine ».

Les disparités en termes de résultats dans la littérature peuvent s'expliquer principalement soit par la non linéarité de l'exposition de change avec l'utilisation de produits de couverture de change complexes, ou à cause des différences de contextes temporels d'une étude à l'autre, les multitudes de variables proxies et finalement la diversité des populations étudiées.

En outre, la littérature financière a accordé plus d'attention à la manière dont la volatilité de change influence la performance financière de l'entreprise internationale :

Premièrement, le mouvement de change affecte les recettes de 1'entreprise de diverses façons dont je cite l'Effet Marge que certains auteurs lient au Pass-Through. Ce dernier concept définie l'intensité de transmission de la variation de change du fournisseur au client à travers un changement du prix de vente. Ainsi un transfert incomplet de la fluctuation signifie une érosion de la marge commerciale.

A ce titre, Hung (1993) a déduit qu'une appréciation durable du dollar de $10 \%$ engendre une diminution du profit des producteurs américains de $6 \%$.

\footnotetext{
${ }^{3}$ Variabilité du taux de change, flux commerciaux, croissance économique : le cas de Madagascar / Razafindramanana Olivasoa Miaranirainy P112
} 
Quant à Mann (1986) a remarqué que 20 à 50\% des variations des taux de change sont intégrés dans les marges des entreprises exportatrices vers les Etats-Unis. De surcroit, Athukorala (1991) juge que les exportateurs coréens intègrent $72 \%$ de la fluctuation de change dans leurs marges dans un délai d'un an.

Idem, Bhattacharya, Karayalcin et Thomakos (2003) ont mis sous la loupe le pass-through des exportations des Etats-Unis vers le Japon. Ils ont eu un bilan mitigé allant de 7\% (pour les machines et les équipements) jusqu'à $100 \%$ (pour le bois). De même, Campa et Goldberg (2005) ont trouvé qu'empiriquement la transmission de la variation de change sur le prix est partielle et se situe entre 0.5 et 1dans les pays de l'OCDE.

Plus récemment, Choudhri et Hakura (2014) ont dévoilé que le coefficient pass-through moyen est de 0.39 chez les exportateurs et de 0.67 chez les importateurs d'un échantillon de 34 pays dont 18 développés et 16 en voie de développement.

En réalité, la dynamique du pass-through est plus complexe car les variations de change ont tendance à s'auto-annuler en revenant au taux d'équilibre selon l'école Keynésienne par conséquent le manager aura du mal à se prononcer sur une éventuelle modification du prix. Ce phénomène s'appelle « Mean reversion ».

D'ailleurs, Baldwin et Krugmann (1989) parlent du Concept d' "hystérésis des échanges internationaux» qui ressemble à une sorte d'inertie ou des facteurs comme la fidélité à la marque rendent le pass-through incomplet ou le retardent. Ils recommandent de négliger dans les études les fluctuations passagères et/ou reversées ainsi que les variations minimes.

Deuxièmement, le mouvement de change peut affecter le volume des ventes de l'entreprise cependant il est moins visible comptablement. A cet égard, Zhang et Kinnucan (2014)ont déduit qu'une hausse de 1\% du Peso Chilien par rapport au Dollar fait baisser la demande américaine du saumon adressée au Chili de $0.13 \%$ c'est-à-dire la volatilité de change est devenue un facteur favorisant la substituabilité entre pays concurrents du marché du saumon. L'effet volume apparait et disparait d'un marché à l'autre car il dépend de la structure concurrentielle et environnementale Froot et Klemperer (1989), Moffett et Karlsen (1994). Ces derniers jugent que dans le Marché des produits non différenciés la distinction se fait essentiellement sur la base des prix en effet une évolution de change considérable qui se transmet au prix de la firme réoriente la demande vers les concurrents si ils réagissent avec opportunisme en gardant ou en baissant leurs prix. A l'inverse, dans un Marché des niches ou la compétitivité se base sur la qualité et la spécialisation l'effet volume est moins prononcé voire inexistant. La situation est presque similaire dans le Marché à parts fixées car l'Etat régule les prix. 
Le mouvement de change peut renverser complètement l'équilibre concurrentiel en attirant un nouveau acteur sur le marché ce qui menace la demande adressée à l'entreprise. Toutefois, Krugman et Baldwin (1987) posent la condition que cette variation de change dépasse le seuil permettant de couvrir « les couts irrécupérables » pour l'entrée au marché.

Quant au Maroc, Achy et Sekkat (2003) ont conclu que le producteur national joue le rôle de " price-taker » en euro surtout dans le secteur agricole et de l'industrie chimique. Autrement dit, les exportateurs marocains ajustent leurs offres vers le marché européen en fonction de l'évolution du taux de change afin de maximiser les profits exprimés en monnaie locale. En effet, si le Dirham se déprécie l'exportateur dégagera une marge supérieure ce qui le pousse à augmenter les volumes exportés soit en détournant une partie de l'offre dédiée au marché domestique vers l'étranger soit en augmentant la production si la capacité le permet. Toutefois, Serge Rey (2006) n'a pas confirmé les incidences de la volatilité réelle de change sur les exportations marocaines et algériennes. En paradoxe, la volatilité de change nominale a des effets positifs pour ces deux pays de façon significative à $10 \%$.

Nous pouvons résumer le mécanisme de fluctuation de change qu'affronte une entreprise exportatrice en ce qui suit :

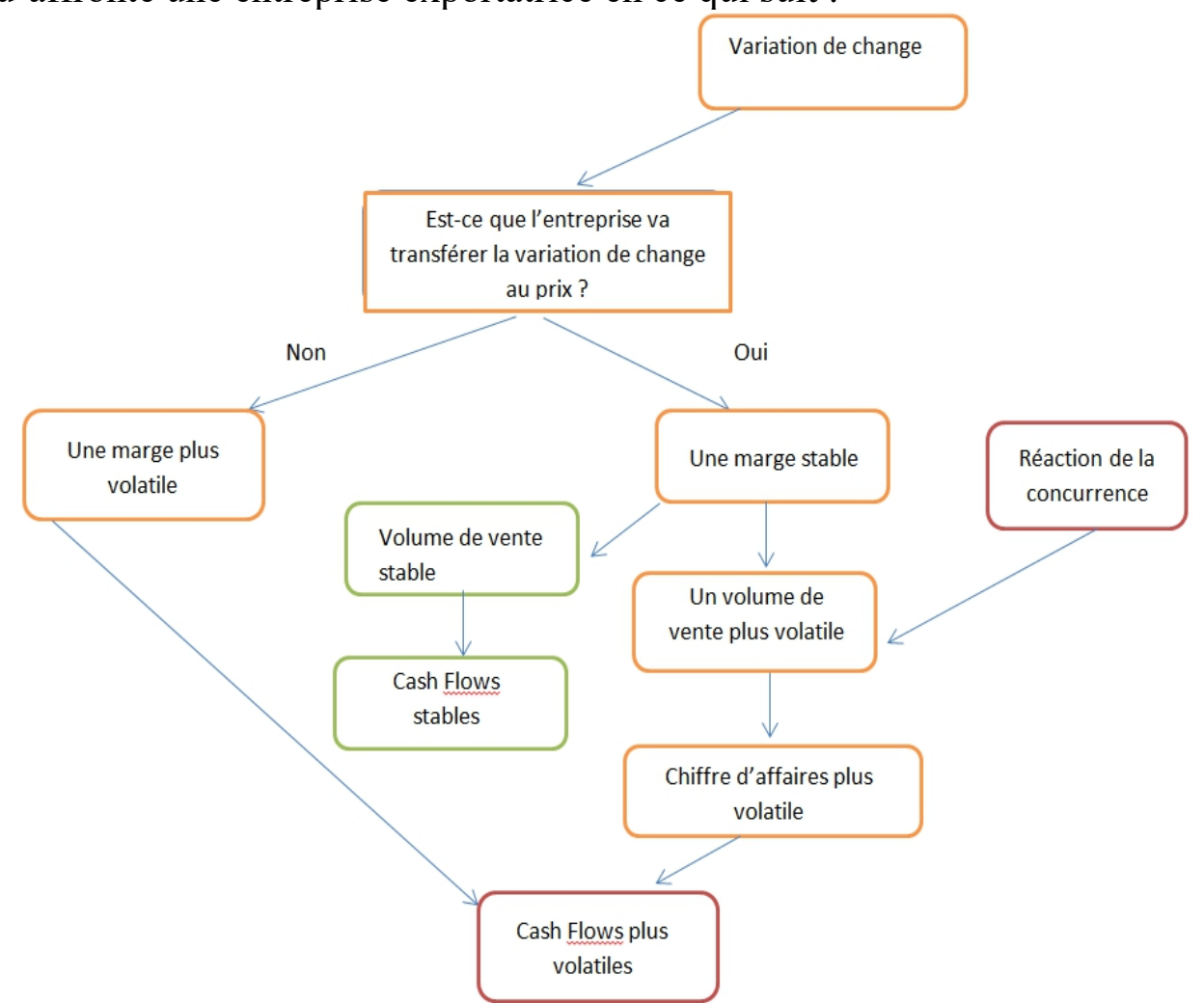

Figure 1: L'exposition du chiffre d'affaire à l'export aux variations de change dans un marché des produits non différenciés Source: Elaboré par l'auteur. 
Troisièmement, la volatilité de change menace parfois les charges de la société via le processus du pass-through surtout si elle procède à des opérations d'importation. La situation se complique davantage si elle combine exportation et approvisionnement depuis l'étranger. A ce titre, Bartram, Dufey et Frenkel (2005) pensent que le cout de production de la filiale s'accroit lors d'une dépréciation de sa devise de façon moindre que l'augmentation du chiffre d'affaires calculé en monnaie de la société mère. En effet, la dépréciation a un effet positif net sur le profit de la société mère.

D'autres cas de figures sont plus complexes comme le lien entre le prix du pétrole et le taux de change. A ce sujet, Valérie Mignon (2009) confirme l'existence de relation positive pour la période de 1986 à 1997. A contrario, le sens de la corrélation devient négatif entre 2002 et 2008. Ce qui logiquement perturbe la facture énergétique de la société.

Quatrièmement, le besoin en fonds de roulement n'est pas à l'abri du risque de change comme l'indique Sandmo (1971) qui stipule qu'un taux de change trop agité amplifie l'incertitude relative aux prix des intrants et du produit final ce qui amène l'entreprise à limiter sa production. De l'autre côté, les partisans du Théorème de la séparation sont convaincu que le taux de change au comptant n'influence plus l'entreprise à ce sujet Holthausen (1979), Wahl et Broll (1995) ont conclu que l'entreprise produit sans se soucier trop de la volatilité lorsqu'un marché de change performant proposant des offres de couverture intéressantes existe. Le modèle de UngernSternberg et Weizsäcker (1990) justifie ce constat par le recours de l'entreprise dans la première phase à la décision de couverture puis elle prend des décisions opérationnelles de production en se référant sur les taux à terme dans la deuxième phase. En revanche, Coté (1994) estime que la couverture au risque de change est fiable uniquement pour les sociétés ayant un cycle d'exploitation court. Néanmoins, les firmes qui prennent un temps considérable entre l'approvisionnement, fabrication et livraison souffriront des produits de change couteux vu le délai exigé. Au Maroc, Alaoui Ismaili et El M'kaddem (2009) n'ont pas soulevé de relation entre cycle d'exploitation et sensibilité à la volatilité de change.

Cinquièmement, Lizondo et Montiel (1989) confirment l'impact d'une dépréciation sur les salaires nominaux. Idem, Nucci et Pozzolo (2010) se sont concentrés sur un panel d'entreprises italiennes ce qui leur a permis de témoigner de l'impact de la variation de change sur l'emploi, le nombre d'heures de travail et les salaires.

Or, l'approche macroéconomique propose une vision complètement différente car elle préconise que le taux de change n'est plus une claire variable exogène ou causale il devint la une conséquence ou encore un simple signe d'un mécanisme plus complexe. Ainsi, un différentiel de productivité croissant en faveur des biens échangeables« industrie... » donne lieu à la fois 
à une augmentation du prix des biens non échangeables "souvent des entreprises domestiques affilées au secteur des services ou de l'artisanat...» et à une appréciation réelle de la monnaie nationale.

L'importance de cette appréciation est en fonction de l'écart du degré de dominance du facteur travail par rapport au facteur capital entre biens échangeables et biens non-échangeables. Ce processus est connu sous « L'effet Balassa-Samuelson (1964)» :

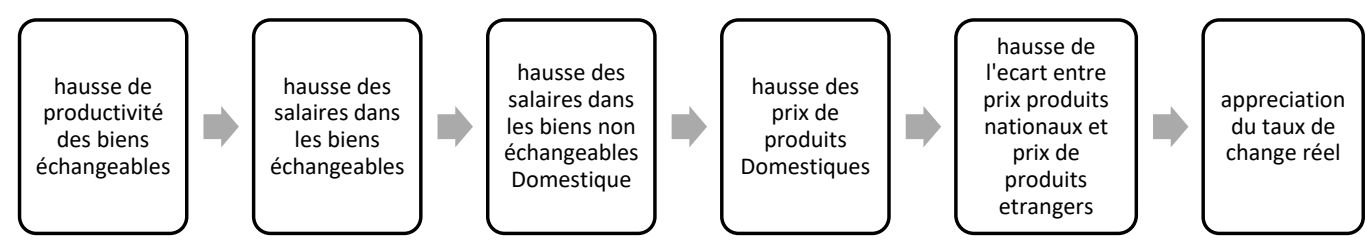

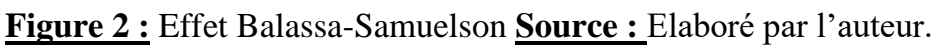

En outre, les entreprises domestiques « qui achète et vend localement » sont également concernées par la problématique de volatilité de change.

A cet égard, Bergbrant, Campbell et Hunter (2014) ont adressé un questionnaire aux 10000 managers de 2458 entreprises dans 55 pays. Ils ont recensé $63 \%$ des firmes domestiques qui ont jugé que le risque de change menace leurs activités. " $80 \%$ des entreprises internationales 》.

Par ailleurs, Martin et Mauer (2003) se sont penchés sur les banques des Etats unis en montrant que $88 \%$ des banques domestiques sont fragiles aux mouvements de change avec un effet du décalage temporel plus prononcé contre $72 \%$ des banques internationales cette différence peut s'expliquer selon Mian (1996), Chow et al. (1997b), Geczy et al. (1997) par l'économie d'échelle et la bonne maitrise du risque de change de cette dernière catégorie.

Le mécanisme reliant volatilité de change et entreprises domestiques ne se limite pas à l'effet Balassa-Samuelson je cite en premier lieu l'exemple de Aggarwal et Harper (2010) qui parlent du cas spécifique des sociétés de distribution régional touchées par la volatilité qui empiriquement achètent et vendent localement mais en réalité leurs fournisseurs nationaux s'approvisionnent de l'étranger. En effet, ces entreprises domestiques ne sont pas au contact direct avec le risque de change car les fournisseurs jouent le rôle d'écran qui peut transmettre la volatilité d'une manière moins visible.

En deuxième lieu, nous signalons l'effet revenu qui surgit quand la balance commerciale se rétablit et les termes d'échange s'améliorent par conséquence le taux de change progresse. Dans ces conditions, l'économie nationale dégage un revenu supplémentaire qui sera déboursé peu ou prou 
dans des produits importés ou dans des biens échangeables. Autrement dit, un revenu additionnel renforce la demande intérieure ce qui se transformera en partie en commande en faveur des entreprises domestiques.

En troisième lieu, l'effet substitution ou les ménages évincent les biens non-échangeables en faveur des biens importés et vice versa selon le mouvement de change ce qui amène De Gregorio et al., (1994) ; Richaud et al., (2003) à déduire qu' "il est généralement admis que l'effet revenu domine l'effet substitution du côté demande, ce qui implique qu'une amélioration des termes de l'échange est plus susceptible d'aboutir à une appréciation du taux de change réel d'équilibre ${ }^{4} \gg$.

\section{Les données et la méthodologie}

Afin d'étudier l'impact de la volatilité du Dirham sur la performance financière des entreprises marocaines nous avons constitué un échantillon de 56 sociétés cotées à la bourse de Casablanca.

Ces firmes doivent répondre à deux conditions d'éligibilité : Premièrement, l'année d'introduction en bourse précède2007 et deuxièmement l'entreprise doit être toujours active en 2016. En effet, nous avons collecté les données financières de 11 à 14 ans en fonction de la disponibilité des communications financières. Nous étions intéressés par toute catégorie d'entreprise « importatrice, exportatrice et domestique » appartenant à tous les secteurs d'activité.

Nous mentionnons que nous avons exclu des sociétés de notre périmètre d'analyse telles que SAMIR, WAFA ASSURANCE et autres pour des raisons qui peuvent biaiser nos résultats comme la situation de crise financière, méthodes comptables incompatibles avec l'objectif de notre étude, présentation non détaillée ou peu claire des états de synthèse ... au total nous avons constitué un échantillon de 56 entreprises marocaines.

Nous avons récolté les états de synthèse grâce au site web de l'Autorité Marocaine du Marché des Capitaux. De plus, nous avons obtenu les taux de change des sources suivants :

\begin{tabular}{|c|c|}
\hline Devise & Base de données \\
\hline Euro et Dollar & Codes générales des impôts \\
\hline $\begin{array}{l}\text { Dollar canadien, Riyal saoudien, } \\
\text { Dirham émirati, Yen, Dinar tunisien, } \\
\text { Dinar algérien, Ouguiya mauritanien } \\
\text { et le dinar libyen }\end{array}$ & $\begin{array}{l}\text { Base de données MANAR du ministère des } \\
\text { finances }\end{array}$ \\
\hline Taux de change effectif réel (REER) & Site web du Fonds Monétaire International \\
\hline $\begin{array}{c}\text { Franc CFA, Won coréen et le Yuan } \\
\text { chinois }\end{array}$ & $\begin{array}{c}\text { Site web } \\
\text { https://www,oanda,com/lang/fr/currency/average }\end{array}$ \\
\hline
\end{tabular}

${ }^{4}$ L'approche microéconomique du taux de change réel d'équilibre : une revue de la littérature théorique, Jean-François Hoarau p422 
Par ailleurs, nous avons examiné plusieurs indices pour déterminer la monnaie des partenaires de l'entreprise à l'instar de l'Office Marocain de la Propriété Industrielle et Commerciale, la plateforme Kerix Export et d'autres sites spécialisés en information économique.

En tenant compte de la nature des données disponibles nous avons privilégié la régression linéaire simple et multiple comme outils d'analyse. En effet, nous avons retenu les équations suivantes :

$$
\begin{gathered}
\Delta R e s=\beta_{0}+\beta_{i} \Delta X_{i}+\varepsilon \\
\Delta R e s=\beta_{0}+\beta_{i} \Delta R E E R+\varepsilon \\
\Delta B F R=\beta_{0}+\beta_{i} \Delta X_{i}+\varepsilon \\
\Delta B F R=\beta_{0}+\beta_{i} \Delta R E E R+\varepsilon \\
\Delta P e r=\beta_{0}+\beta_{i} \Delta X_{i}+\varepsilon \\
\Delta P e r=\beta_{0}+\beta_{i} \Delta R E E R_{i}+\varepsilon
\end{gathered}
$$

Avec :

- Res : résultat courant

- BFR : besoin en fonds de roulement d'exploitation

- Per : charges de personnel

- $\mathrm{X}_{\mathrm{i}}$ : la moyenne annuelle des taux de change à l'incertain du partenaire « client ou fournisseur»

- REER : taux de change effectif réel

- $\varepsilon$ : résidus

Nous signalons que pour certains cas très rares nous avons utilisé le résultat net au lieu du résultat courant à cause de l'indisponibilité des données ou de la nature du secteur d'activité :

$$
\begin{gathered}
\Delta \text { Res }=\beta_{0}+\alpha_{i} \Delta \text { Per }+\beta_{i} \Delta X_{i}+\varepsilon \\
\Delta \text { Res }=\beta_{0}+\alpha_{i} \Delta \text { Per }+\beta_{i} \Delta R E E R+\varepsilon
\end{gathered}
$$

Dans la seconde équation nous avons introduit les $\Delta$ Per comme variable de control pour mieux cerner l'effet de la volatilité de change.

Nous précisons que nous avons adopté une marge d'erreur de 10\%. De plus, nous avons utilisé le test d'autocorrélation des erreurs de Durbin et Watson, le Test de colinéarité de Klein et le test d' homoscédasticité de Koenker. 
4. Résultats et discussions

L'essentiel des résultats obtenus se manifeste dans le tableau ci-dessous :

\begin{tabular}{|c|c|c|c|c|c|c|c|}
\hline $\mathrm{N}$ & Entreprise & $\begin{array}{c}\text { Variable } \\
\text { dépendant } \\
\mathrm{e}\end{array}$ & $\begin{array}{c}\mathrm{R} \\
\text { ADJUS } \\
\text { TED }\end{array}$ & $\begin{array}{c}\text { DEVIS } \\
\text { E }\end{array}$ & $\begin{array}{l}\text { Standardi } \\
\text { zed } \\
\text { Coefficie } \\
\text { nts }\end{array}$ & Activité & Secteur \\
\hline & AUTONEJMA & $\Delta$ Per & $84 \%$ & Euro & $(0.94)$ & \multirow[t]{2}{*}{ Importateur } & \multirow[t]{2}{*}{ Distribution } \\
\hline 1 & & & & Won & $(0.03)$ & & \\
\hline 2 & MICRODATA & Résultat & $21 \%$ & REER & $(0.54)$ & Domestique & Informatique \\
\hline 3 & COLORADO & $\triangle \mathrm{BFR}$ & $31 \%$ & REER & $-0,62$ & $\begin{array}{l}\text { Importateur/ } \\
\text { Exportateur }\end{array}$ & Chimie \\
\hline 4 & $\begin{array}{c}\text { FENIE } \\
\text { BROSSETTE }\end{array}$ & $\Delta \mathrm{BFR}$ & $29 \%$ & Dollar & $-0,6$ & $\begin{array}{l}\text { Importateur/ } \\
\text { Exportateur }\end{array}$ & Distribution \\
\hline \multirow[b]{2}{*}{5} & \multirow[t]{2}{*}{ INVOLYS } & Résultat & $22 \%$ & REER & $-0,54$ & \multirow[t]{2}{*}{ Exportateur } & \multirow[t]{2}{*}{ Informatique } \\
\hline & & $\Delta \mathrm{BFR}$ & $38 \%$ & CFA & $(-0,66)$ & & \\
\hline \multirow[b]{3}{*}{6} & \multirow[t]{3}{*}{ LAFARGE } & Résultat & $48 \%$ & REER & 0,73 & \multirow{3}{*}{$\begin{array}{l}\text { Importateur/ } \\
\text { Exportateur }\end{array}$} & \multirow{3}{*}{$\begin{array}{l}\text { Matériaux de } \\
\text { Construction }\end{array}$} \\
\hline & & $\triangle \mathrm{BFR}$ & $32 \%$ & Euro & $-8,72$ & & \\
\hline & & $\triangle \mathrm{BFR}$ & & CFA & $(-8,64)$ & & \\
\hline 7 & $\begin{array}{c}\text { MAROC } \\
\text { LEASING }\end{array}$ & Résultat & $58 \%$ & $\begin{array}{c}\text { Euro }+ \\
\Delta \text { Per }\end{array}$ & 0,51 & Domestique & Financement \\
\hline \multirow[b]{2}{*}{8} & \multirow[t]{2}{*}{ NEXANS } & \multirow[t]{2}{*}{$\Delta \mathrm{BFR}$} & \multirow[t]{2}{*}{$62 \%$} & Dollar & $(3,22)$ & \multirow{2}{*}{$\begin{array}{l}\text { Importateur/ } \\
\text { Exportateur }\end{array}$} & \multirow{2}{*}{$\begin{array}{l}\text { Equipements } \\
\text { Electroniques } \\
\text { et Electriques }\end{array}$} \\
\hline & & & & CFA & $(-3,25)$ & & \\
\hline \multirow[b]{2}{*}{9} & \multirow{2}{*}{$\begin{array}{l}\text { CARTIER } \\
\text { SAADA }\end{array}$} & \multirow[t]{2}{*}{ Résultat } & \multirow[t]{2}{*}{$54 \%$} & EURO & 4,79 & \multirow[t]{2}{*}{ Exportateur } & \multirow{2}{*}{$\begin{array}{l}\text { Agroalimentai } \\
\text { re }\end{array}$} \\
\hline & & & & Dollar & 5,12 & & \\
\hline \multirow[b]{3}{*}{10} & \multirow[t]{3}{*}{ TASLIF } & Résultat & $28 \%$ & REER & 0.58 & \multirow[t]{3}{*}{ Domestique } & \multirow[t]{3}{*}{ Financement } \\
\hline & & Résultat & $65 \%$ & $\begin{array}{c}\text { Dollar+ } \\
\Delta \text { Per }\end{array}$ & $-0,33$ & & \\
\hline & & $\Delta$ Per & $23 \%$ & REER & $-0,55$ & & \\
\hline & \multirow[t]{2}{*}{ SMI } & \multirow[t]{2}{*}{$\triangle \mathrm{BFR}$} & \multirow[t]{2}{*}{$59 \%$} & YUAN & $(-1,18)$ & \multirow[t]{2}{*}{ Exportateur } & Mines \\
\hline 11 & & & & EURO & $(-0,65)$ & & \\
\hline & STOKVIS & Résultat & $38 \%$ & EURO & $(0,73)$ & Importateur & Distribution \\
\hline 12 & NORD & Résultat & & YEN & $(0,4)$ & & \\
\hline & AFRIQUE & $\Delta \mathrm{BFR}$ & $34 \%$ & EURO & $(0,64)$ & & \\
\hline 13 & SOTHEMA & $\triangle \mathrm{BFR}$ & $41 \%$ & $\begin{array}{c}\text { DINAR } \\
\text { TUNIS } \\
\text { IEN }\end{array}$ & 0,53 & $\begin{array}{l}\text { Importateur/ } \\
\text { Exportateur }\end{array}$ & $\begin{array}{c}\text { Pharmaceutiqu } \\
\text { e }\end{array}$ \\
\hline & SONASID & Résultat & $31 \%$ & REER & 0,62 & $\begin{array}{l}\text { Importateur/ } \\
\text { Exportateur }\end{array}$ & $\begin{array}{l}\text { Matériaux de } \\
\text { Construction }\end{array}$ \\
\hline & & $\triangle \mathrm{BFR}$ & $41 \%$ & EURO & 0,9 & & \\
\hline 14 & & & & $\begin{array}{l}\text { OUGU } \\
\text { IYA }\end{array}$ & 0,75 & & \\
\hline & SNEP & Résultat & $30 \%$ & EURO & $(-8,24)$ & Importateur/E & Chimie \\
\hline 15 & & & & CFA & $(-8,4)$ & xportateur & \\
\hline
\end{tabular}

Les parenthèses () signifient que le signe ne correspond pas à la littérature.

$\underline{\text { Table } 1}$ : Impact de la volatilité de change sur la performance des entreprises cotées. 
A l'issue de cette analyse nous déduisons que la problématique de la volatilité de change concerne 15 sociétés cotées ce qui représente $26 \%$ de notre échantillon. Par ailleurs, le profil de ces entreprises indique que la variation de change ne se limite pas à un seul segment car elle touche le secteur primaire, secondaire et tertiaire.

En revanche, nous avons remarqué une sensibilité particulière de certaines branches comme la chimie, la distribution et des matériaux de construction. Il n'est pas fortuite que la concurrence acharnée dans ces domaines se pratique sur la base des prix ce qui amplifie l'effet de la volatilité de change. Toutefois, nous mentionnons l'insensibilité des banques à caractère internationales ceci s'explique soit par une bonne gestion du risque de change ou par l'inadéquation du modèle économétrique utilisé. De plus, nous évoquons la grande disparité au niveau du degré d'influence qui commence par « la société Taslif » jusqu'au pic « l'entreprise Autonejma ».

En sus, nous signalons la dominance des firmes internationales touchées par la variation de change par rapport aux entreprises domestiques « 13 contre 2 ». L'exposition de la première catégorie est importante car ces sociétés ont des liens directs et visibles avec l'étranger contrairement aux entreprises domestiques qui entretiennent des relations indirects avec l'extérieur.

Dans ce volet, nous citons les deux sociétés de financement « Taslif et Maroc leasing » qui n'ont pas de rapport clair à l'international. Nous pensons que l'exposition significative du premier cas peut s'expliquer par l'effet volume puisque Taslif propose des crédits de consommation souvent liés à des produits et services importés « EX : automobile ...».

De même, Maroc leasing offre principalement des crédits-baux aux entreprises pour financer l'acquisition des engins industriels importés. Dans ce genre de cas le client joue le rôle d'écran entre le fournisseur et la société de financement.

En outre, nous avons constaté que 9 parmi 13 devises testées ont un impact considérable sur la performance de l'entreprise. Ceci montre la multiplication des partenaires économiques du Maroc et l'entrée du pays dans la dynamique de la mondialisation. Cependant, les monnaies internationales occupent une place importante étant donné qu'elles touchent la majorité écrasante des entreprises du tableau en haut.

Rappelons que nous avons utilisé la cotation du Dirham à l'incertain, nous avons remarqué que 9 entreprises ont un résultat significativement sensible aux variations de change soit $16 \%$ de notre échantillon. Toutefois, juste 6 d'entre elles ont des signes de coefficient d'exposition conformes à la littérature.

Idem, nous avons observé que 9 sociétés possèdent une variation du besoin en fonds de roulement significativement liée à la volatilité de change 
en revanche juste 6 d'entre elles ont des signes qui correspondent à l'aspect théorique. Ceci montre que la pratique du termaillage n'est pas très répondue au sein de notre échantillon.

Nous mentionnons l'absence de corrélation entre les charges de personnel et les variations de change sauf pour un seul cas isolé. Ce constat reflète la rigidité des salaires et le manque de souplesse du code du travail au Maroc.

Nous pensons que l'origine des faibles résultats significatifs réside d'une part dans les problèmes statistiques rencontrées dont la difficulté de faire appel à des variables de control ceci découle du nombre limité des données collectées « une dizaine d'années ». D'autre part, les divergences provenant des méthodes comptables nationales et internationales peuvent altérer les résultats.

En sus, le régime de change fixe pratiqué au Maroc dans le but d'adoucir les chocs peut réduire les effets de la volatilité de change. De plus, une forte utilisation des produits de couverture de change rendent l'exposition non-linéaire.

\section{Conclusion}

Dans le présent article nous avons discuté dans un premier temps les effets de la volatilité de change sur la performance des entreprises cotées à la bourse de Casablanca du point de vue théorique. Par la suite, nous avons présenté notre modèle qui englobe les observations de la dernière décennie. En sus, nous avons exposé les résultats significatifs obtenus.

A la lumière de ce qui précède nous déduisons que l'effet de la volatilité de change sur le résultat courant et le besoin en fonds de roulement est palpable au Maroc. Néanmoins, l'étendu et l'intensité de l'impact des variations de change ne correspond pas au degré cité dans la littérature. De surcroît, les tests menés confirment l'absence d'une relation causale contemporaine entre les charges de personnel et la variation de change. D'ailleurs, Nous avons conclu que le risque de change est de type idiosyncratique.

En somme, nous recommandons aux autorités boursières et comptables d'uniformiser les normes de présentation des états financiers. Ainsi, nous incitons les entreprises sensibles à la volatilité de change à diffuser des notes d'information relatives à ce risque et à améliorer leurs managements à ce sujet. Par ailleurs, nous préconisons des études académiques qui simulent le comportement du Dirham après la libéralisation. 


\section{References:}

1. Achy, L., Sekkat, K. (2003).The European Single Currency and MENA's Exports to Europe, Review of Development Economics. 7 ( 4) : 563-582.

2. Aggarwal, R., Harper, T. (2010). Foreign exchange exposure of "domestic" corporations, Journal of International Money and Finance. 29(8) : 1619-1636.

3. Bartram et al. (2005). A primer on the exposure of non-financial corporations to foreign exchange rate risk. Journal of Multinational Financial Management. $15: 394-413$.

4. Bartram, M. (2008). What lies beneath: Foreign exchange rate exposure, hedging and cash flows. Journal of Banking\& Finance. 32(8) :1508-1521.

5. Bergbrant, K. et al. (2014). Firm-Level Competition and Exchange Rate Exposure: Evidence from a Global Survey of Firms. Financial Management. 4 : 885 - 916.

6. Chnaina, K.(2013). Les Effets de la Variabilité du Taux de Change Réel sur le Commerce Extérieur. Cas de la Tunisie. Thèse de Doctorat en sciences économiques :Université de Pau et des Pays de l'Adour.

7. Choudhri, U., Hakura, S.(2014). The Exchange Rate Pass-Through to Import and Export Prices: The Role of Nominal Rigidities and Currency Choice. Journal of International Money and Finance. 51 : $1-25$.

8. Drine, I., Rault, C.(2007). Fluctuations de change et performances economiques, Brussels Economic Review - Cahiers Economiques De Bruxelles.

9. Du et al. (2012). Measuring currency exposure with quantile regression, Review of Quantitative Finance and Accounting. 41(3): 549-566.

10. Fontaine, P. (2009). Marché des changes. Pearson Education France. 127-196.

11. Häberle, R. (1999). Risque de change, entreprise et investisseur. Le cas du marché suisse des actions. Thèse de Doctorat en sciences économiques : Université de Fribourg.

12. Hoarau, J. (2009). L'approche microéconomique du taux de change réel d'équilibre : une revue de la littérature théorique, L'Actualité économique. Revue d'analyse économique, vol 85, n²4, 403-423.

13. Ismaili, A., M'kaddem A. (2009). Variations des taux de change et facteurs de sensibilité des entreprises exportatrices marocaines. Critique économique. 23 :35-65. 
14. Krapl, A., O'Brien, J. (2015). Direct versus indirect regression estimates of foreign exchange cash flow exposure. International Review of Financial Analysis. 37 : 103-112.

15. Martin D., Mauer J. (2003). Exchange rate exposures of US banks: A cash flow-based methodology. Journal of Banking \& Finance. 27 : 851-865.

16. Martin, D., Mauer, J. (2003). Transaction versus economic exposure: which has greater cash flow consequences?. International Review of Economics and Finance. 12 (4) : 437-449.

17. Miaranirainy, O. (2015).Variabilité du taux de change, flux commerciaux, croissance économique : le cas de Madagascar. Thèse de Doctorat en sciences économiques : Universite de Pau et des Pays De L'adour.

18. Mignon,V. (2009). Les liens entre les fluctuations du prix du pétrole et du taux de change du dollar. Revue d'économie financière. 94 (1) : 187-195.

19. Moffett, H., Karlsen, K. (1994). Managing Foreign Exchange Rate Economic Exposure. Journal of International Financial Management Accounting. 5(2) : 157-175.

20. Rey S. (2006). Effective exchange rate volatility and mena countries' exports to the eu. Journal of economic development. 31(2) : 27-45.

21. Sabiston R. (2001). Le pass-through du taux de change : un survol de la littérature. L'Actualité économique. 77(3) : 425-454.

22. Zhang D. et Kinnucan W. (2014). Exchange Rate Volatility and US Import Demand for Salmon. Marine Resource Economics. 29(4) : 411430. 